\title{
Análise do padrão RDA: um estudo aplicado em teses e dissertações em literatura e cinema
}

\author{
Analysis of the RDA standard: a applied study on theses and dissertation in literature \\ and cinema
}

Raquel Bernadete Machado, Ana Maria Pereira

Universidade Federal de Santa Catarina

RESUMO: O Resource Description and Access (RDA) é a nova diretriz para a catalogação desenvolvida para substituir o Código de Catalogação Anglo-Americano, segunda edição (AACR2), com a finalidade de melhorar a recuperação da informação. Nesse sentido, a presente pesquisa objetiva analisar como os registros bibliográficos de teses e dissertações das áreas de literatura e cinema serão modificados com os padrões do código RDA. A pesquisa caracteriza-se como qualitativa aplicada de caráter exploratório e descritivo, e utiliza como método o estudo de caso. Os resultados do estudo apontaram que a descrição bibliográfica apresenta modificações significativas correspondentes ao formato, conteúdo e mídia de um recurso. Com base na análise dos registros e dos resultados obtidos, pode-se concluir que o catalogador precisa despender mais atenção, conhecimento e tempo na sua prática, o que sugere a esse profissional a necessidade de educação continuada.

PALAVRAS-ChaVE: RDA. Descrição e Acesso de Recursos. Regras de Catalogação. Registro bibliográfico.
ABSTRACT: The Resource Description and Access (RDA) is the new guideline for cataloging developed for to replace the Anglo-American Cataloging Rules, Second Edition (AACR2) with the purpose of to improve information retrieval. In this sense, this research aims to analyze how bibliographic records of theses and dissertations on areas of literature and cinema will be modified with the RDA standards. The research is characterized as qualitative applied exploratory and descriptive and used as method the case study. The results of the study pointed out that the bibliographic description presents significant changes in referring to the format, content and media of a resource. Based on the analysis of the records and the results obtained, it can be concluded that the cataloger needs to spend more attention, knowledge and time in your practicing, suggesting to this professional the need for continuing education.

KEYWORDS: RDA. Resource Description and Access. Cataloging rules. Bibliographic record

\author{
Correspondência \\ Raquel Bernadete Machado \\ Universidade Federal de Santa Catarina. \\ Florianópolis, SC. \\ Email: raquelfloripa@gmail.com \\ ORCID: http://orcid.org/0000-0002-1619-6875
}

JITA: IA. Cataloging, bibliographic control. 


\section{INTRODUÇÃ̃}

O RDA é a nova diretriz para a catalogação desenvolvida para substituir o Código de Catalogação Anglo-Americano, segunda edição (AACR2), e tem como intuito melhorar a recuperação da informação. Nesse sentido, o presente artigo relata os resultados obtidos na aplicação do RDA em teses e dissertações nas áreas de literatura e cinema, da Universidade Federal de Santa Catarina (UFSC). O objetivo geral do estudo foi analisar como os registros bibliográficos serão modificados com os novos padrões do RDA. Dessa forma, elaboraram-se registros bibliográficos de teses e dissertações no padrão RDA para comparar com o padrão AACR2, bem como foram identificados e propostos elementos consistentes para a descrição dos recursos conforme o novo padrão. Ademais, analisaram-se os registros bibliográficos, especificamente no que se refere às entidades-relacionamentos.

A produção científica das teses e das dissertações nas áreas de literatura e cinema é uma rica fonte de expressões, pois são áreas inter-relacionadas e que apresentam formas diferentes de uma mesma obra. Tais documentos, que abordam os dois temas - literatura e cinema, - apresentam em seu conteúdo análises de outras obras, como por exemplo, um livro adaptado para o cinema e que teve tradução para outra língua.

Por outro lado, há uma conexão bastante distinta entre diferentes obras e que não está registrada nos atuais catálogos de bibliotecas, pois as informações apresentadas nesses catálogos são individuais para cada obra e não ocorre uma ligação entre elas. Assim, torna-se relevante conhecer as limitações nos campos de descrição de um recurso informacional.

Após revisão de literatura e estudos a respeito do tema, verificou-se que os atuais sistemas gerenciadores de acervos bibliográficos não apresentam opções para incluir vínculos entre obras diferentes, pois os campos disponíveis nesses softwares são limitados ao descrever informações de natureza física do documento e alguns descritores de conteúdo temático.

O estudo acerca dos padrões RDA é importante na medida em que se propõe analisar os principais aspectos na adoção desse novo padrão. É relevante verificar igualmente quais as dificuldades encontradas em uma possível implementação das novas regras e dos padrões aplicados aos registros catalográficos. Tal verificação proporcionará benefícios tanto aos usuários das bibliotecas como aos catalogadores no seu fazer diário.

A relevância deste estudo reside ainda na contribuição teórica e prática para a área da Biblioteconomia, por discutir e abordar questões a propósito da temática da catalogação, analisando o código RDA, assunto atual em discussão dos grupos e dos eventos realizados pelos profissionais da área.

A estrutura deste trabalho é composta por uma breve fundamentação teórica acerca da catalogação, do RDA e dos requisitos funcionais para registros bibliográficos. Em seguida,

\begin{tabular}{|c|c|c|c|c|c|}
\hline (C) RDBCI: Rev. Digit. Bibliotecon. Cienc. Inf. & Campinas, SP & v.15 & n.1 & p. $130-147$ & jan./abr. 2017 \\
\hline
\end{tabular}


são mostrados e analisados dois exemplos de aplicação do RDA. Por fim, apresentam-se as considerações finais.

\section{FUNDAMENTAÇÃO TEÓRICA}

A base teórica deste artigo fundamenta-se na catalogação e sua finalidade, bem como as atuais tendências da área. O modelo conceitual que recomenda requisitos funcionais para registros bibliográficos é a base do novo padrão RDA. Assim, por meio de uma breve revisão de literatura são expostos os principais conceitos e objetivos desses temas.

\subsection{Catalogação}

Em uma unidade de informação, a catalogação proporciona o acesso a todo tipo de documento por meio dos registros bibliográficos dos catálogos. Esses registros são elaborados com base nos padrões dos códigos de catalogação, que, por sua vez, foram estabelecidos de acordo com as características gerais e peculiares dos documentos.

Em bibliotecas, garantir o acesso à informação é uma atividade essencial que demanda diversos processos técnicos para melhor gerir a massa documental de uma unidade de informação em seus diversos suportes e, atualmente, com ênfase no ambiente digital. Além da classificação e da indexação, entre tais processos, destaca-se a catalogação, cuja finalidade principal é a recuperação da informação pelos usuários.

A catalogação necessita de alguns instrumentos para o processamento da informação. Entre eles, cita-se o Anglo-American Cataloguing Rules, $2^{\text {a }}$ edição, conhecido como AACR2; em português é denominado como Código de Catalogação Anglo-Americano (2005). O referido código estipula regras para a descrição das informações contidas nos documentos e fornece pontos de acesso a fim de representar os itens informacionais de um acervo por meio dos catálogos.

Assim, os catálogos são criados para atender às necessidades informacionais dos usuários. Entende-se por usuário todos os indivíduos que utilizam o catálogo para buscar algum tipo de informação e inclui a comunidade universitária, além dos bibliotecários de outras instituições e a socidade em geral.

Destaca-se que, para definir as reais necessidades informacionais desses usuários seria necessário desenvolver um estudo mais aprofundado. Contudo, entende-se que um catálogo deve atender minimamente à busca, à recuperação da informação e a uma demanda informacional. Todavia, essa demanda tem se modificado substancialmente com o uso das tecnologias da informação e comunicação.

Em bibliotecas universitárias, as teses e as dissertações configuram-se como documentos de grande visibilidade dessas unidades de informação, pois resultam de 
pesquisas e de estudos realizados pela comunidade acadêmica e que são também de interesse da sociedade.

Alguns tipos de obra relacionam-se com outras por meio da temática; outras, por intermédio dos autores e colaboradores, como é o caso das áreas de literatura e cinema.

As referidas áreas de conhecimento, literatura e cinema apresentam uma relação intermidiática que acontece de diferentes modos, conforme apontado por Müller (2008), em um universo midiático bastante amplo e que inclui diferentes tipos de processos (mutação, transformação, transferência, tradução, adaptação, citação, hibridação), entre essas duas mídias, e, ainda, em relação a outras mídias.

Além disso, é bastante comum, nessas áreas, obras que se transformam em outras, como é o caso das adaptações e das traduções. Com isso, uma mesma obra pode apresentar diferentes nuances, olhares e percepções. No entanto, tais interligações não são evidentes nos atuais catálogos.

Justifica-se a escolha destas duas temáticas - literatura e cinema - por identificar-se previamente que tais áreas apresentam relacionamentos bibliográficos entre obra, expressão e manifestação. Ou seja, nas teses e nas dissertações da área de literatura, e que abordam também o cinema, encontram-se relacionamentos entre autores e outras obras.

O RDA objetiva melhorar a recuperação da informação, descrevendo detalhadamente os recursos informacionais e suas relações com outros recursos. O novo padrão para a catalogação descritiva está pautado no modelo conceitual denominado Functional Requirements for Bibliographic Records (FRBR).

\subsection{Requisitos funcionais para registros bibliográficos (FRBR)}

Após longo período de estudos acerca de padrões adotados na catalogação, um grupo designado pela International Federation of Library Associations (IFLA) publicou, no final da década de 1990, um relatório sobre requisitos funcionais que servissem de referencial para a criação de registros bibliográficos. Durante oito anos, discutiram-se esses requisitos e, em 1998, publicou-se o relatório final denominado FRBR que, traduzido para o português, significa Requisitos Funcionais para Registros Bibliográficos.

O modelo conceitual FRBR, de acordo com Tillett (2003, p. 2), é uma proposta “[...] para todos os tipos de materiais e tarefas de usuários associados com os recursos bibliográficos [...]”. A essência dos FRBR está na tríade composta pelas entidades, atributos e relações encontrados no universo bibliográfico.

O modelo propõe-se a "[...] servir como um ponto de partida para uma série de estudos [...]" que seja base para os especialistas envolvidos com a catalogação e o 
desenvolvimento de "[...] sistemas de apoio à criação, gestão e utilização de dados bibliográficos.” (INTERNATIONAL FEDERATION OF LIBRARY ASSOCIATIONS AND INSTITUTIONS, 2009, p. 5, tradução nossa).

De acordo com Moreno e Medeiros (2008, p. 140) "O modelo FRBR é baseado no modelo computacional Entidade-Relacionamento [...]". A ideia, segundo as autoras, é "[...] repensar o objeto bibliográfico." O foco do modelo FRBR está relacionado com os três elementos básicos - entidades, atributos e relações - e, portanto, é o principal ponto a ser considerado. Le Boeuf, Tillett e Riva (2007, p. 2) definem os três componentes da seguinte maneira:

Um número de classes gerais (“entidades”) de coisas que são julgadas relevantes no contexto específico de um catálogo de biblioteca, seguidas de características ("atributos") que pertencem a cada uma dessas classes gerais, e os relacionamentos que podem existir entre instâncias dessas várias classes.

Assim, as entidades são "coisas" que podem ser entendidas como "[...] objetos-chaves de interesse para os usuários de dados bibliográficos." (INTERNATIONAL FEDERATION OF LIBRARY ASSOCIATIONS AND INSTITUTIONS, 2009, p. 13, tradução nossa). As entidades relacionam-se com todos os elementos do mundo bibliográfico, como uma obra clássica, música, pessoa, conceito, lugar e outros elementos.

De acordo com o relatório do FRBR (INTERNATIONAL FEDERATION OF LIBRARY ASSOCIATIONS AND INSTITUTIONS, 2009), os atributos referem-se ao conjunto de características dessas entidades, ou seja, são as informações pelas quais um usuário procura uma entidade em um catálogo, como por exemplo, título, data, autor e local de publicação. Mey e Silveira (2009, p. 18) observam que os atributos podem desdobrar-se nos conhecidos elementos do tratamento descritivo. Os atributos, desse modo, incluem características físicas e também temáticas.

O último elemento da tríade que compõe o modelo FRBR são as relações que "[...] servem como veículo para descrever a ligação entre uma entidade e outra [...]” , e, assim, configuram-se como o meio de ajudar o usuário a "navegar" no universo bibliográfico de um catálogo ou base de dados (INTERNATIONAL FEDERATION OF LIBRARY ASSOCIATIONS AND INSTITUTIONS, 2009, p. 55, tradução nossa).

As principais entidades do modelo FRBR são obra, expressão, manifestação e item. A obra é considerada a criação intelectual de um autor que possui um conteúdo; a expressão é a realização da obra, ou seja, é como se expressa o conteúdo intelectual; a manifestação é a concretização da expressão, incluindo o conteúdo intelectual em uma forma física; o item, por sua vez, é o exemplar individual de uma manifestação (MEY; SILVEIRA, 2009; OLIVER, 2011). 
Essas quatro entidades apresentam uma inter-relação conforme mostra a Figura 1. Nela é possível visualizar que uma obra é realizada por meio de uma expressão contida em uma manifestação, que por sua vez é exemplificada por um item.

Figura 1. Modelo FRBR

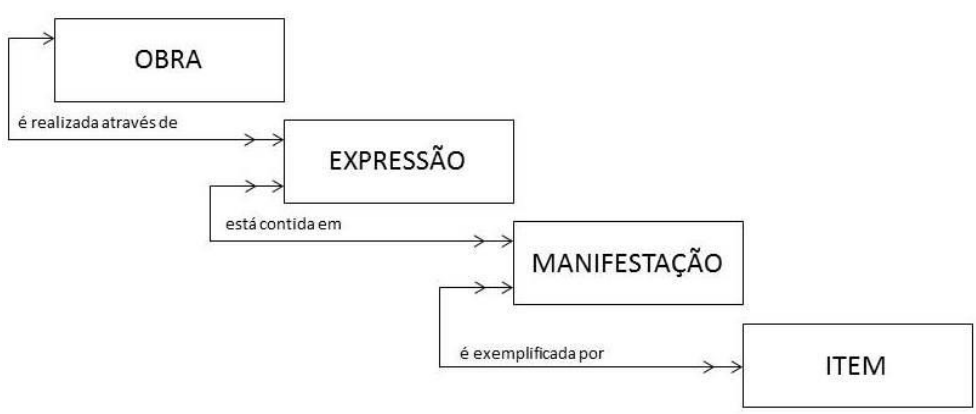

Fonte: International Federation of Library Associations and Institutions (2009, p. 14, tradução nossa).

Nessa concepção, pode-se compreender o modelo conceitual da seguinte forma: a obra "Romeu e Julieta", de William Shakeaspeare, foi realizada originalmente por intermédio da expressão de um texto. Esse texto está contido na manifestação de um livro cujo título é Romeu e Julieta. O exemplar desse livro que determinada biblioteca contém é o item da obra criada pelo autor.

O modelo FRBR mostra como as entidades relacionam-se entre si, como por exemplo, um autor é associado com uma instituição, que, por sua vez está associada a um local geográfico, e assim por diante, formando uma espécie de rede de relacionamentos.

Os FRBR embasaram também a criação das novas diretrizes e do novo padrão RDA, os quais permitem uma integração do universo bibliográfico por meio dos diversos tipos de relacionamentos dos elementos envolvidos como obras, autores e assuntos.

\subsection{Resource Description and Access (RDA)}

O processo de catalogação é realizado com base nos padrões e nas regras estipulados nos códigos de catalogação. O AACR2 é o código utilizado na maioria das unidades de informação. No entanto, em decorrência do avanço tecnológico e dos variados formatos nos quais a informação é disponibilizada, verificou-se que esse padrão não mais atendia ao novo cenário.

Em geral, os códigos de catalogação refletem a organização das bibliotecas e as tecnologias das décadas de setenta e oitenta, e foram pensados para os catálogos em ficha e materiais impressos. Decorridos mais de trinta anos, o contexto informacional contemporâneo modificou-se substancialmente (PICCO; ORTIZ REPISO, 2012).

De acordo com Assumpção e Santos (2013, p. 204), o AACR2 “[...] tornou-se defasado, pois sua estrutura está voltada aos catálogos com descrições em papel, muitas

\begin{tabular}{|c|c|c|c|c|c|}
\hline (C) RDBCI: Rev. Digit. Bibliotecon. Cienc. Inf. & Campinas, SP & v. 15 & n.1 & p. $130-147$ & \\
\hline
\end{tabular}


vezes, no formato de fichas." Dessa forma, houve necessidade de se pensar esse novo cenário, no qual a comunidade bibliotecária representada pela IFLA propôs um novo código denominado RDA em substituição às desatualizadas regras de catalogação do AACR2 revisado.

A partir das revisões do AACR2, surgiu a necessidade de uma nova abordagem das regras de catalogação que contemplasse também os recursos tecnológicos. Assim, segundo Assumpção e Santos (2009, p. 2), “[...] adotou-se então a ideia de um padrão estruturado para o ambiente digital [...]" com a finalidade de fornecer diretrizes e instruções para a descrição e acesso de recursos digitais e analógicos. Fundamentado nos modelos conceituais, desenvolveu-se o RDA com base em uma estrutura teórica, voltada para o ambiente digital, sendo mais abrangente que o AACR2 (OLIVER, 2011).

O RDA foi concebido pelo Joint Steering Committee (JSC) e contou com a participação de diversos países, entre eles Austrália, Canadá, Estados Unidos e Inglaterra. Sua publicação oficial ocorreu em 2010 e trouxe significativas diferenças em relação ao seu antecessor AACR2, entre elas: a nova terminologia, a estrutura mais abrangente, o não uso de abreviaturas e a inclusão de novos campos para a descrição física dos recursos.

Além de diferenciar-se do AACR2, a característica mais evidente do RDA é que esse código, apoiado pelo modelo conceitual entidade-relacionamento, busca especificar as interligações com as várias entidades de um item, como uma rede que conecta as diversas relações existentes entre obra, expressão, manifestação e item. O novo código foi concebido, baseando-se nos FRBR que, de acordo com Assumpção e Santos (2009), permitem ao RDA flexibilidade, extensibilidade e adaptabilidade necessárias aos diferentes ambientes tecnológicos.

No que se refere aos registros bibliográficos, Oliver (2011) afirma que os dados no padrão RDA, isoladamente, não apresentarão melhorias quanto à navegação e exibição, pois dependem de mecanismos e interfaces de busca bem planejados. O grande desafio, na visão de Picco e Ortiz Repiso (2012), é sensibilizar a comunidade informática para que sejam desenvolvidos softwares que atendam às características definidas pelo novo padrão de catalogação.

O código de catalogação RDA é definido em sua introdução e citado por Oliver (2011, p. 1) como "[...] um conjunto de diretrizes e instruções sobre a formulação de dados que sirvam de apoio ao descobrimento de recursos." A autora acrescenta igualmente que o novo código "[...] consiste num conjunto de instruções práticas, que, no entanto, baseia-se numa estrutura teórica que define a forma, a estrutura e o conteúdo desta nova norma”. Essa estrutura teórica está associada aos FRBR nos quais o RDA está fundamentado. 
A nova diretriz apresenta uma estrutura ampla com instruções para descrever atributos e relacionamentos de um recurso, e é disponibilizada pela ferramenta on-line RDA Toolkit (2010), viabilizada por meio de assinatura anual com acesso eletrônico via web.

\section{MATERIAIS E MÉTODOS}

O presente estudo é caracterizado como pesquisa qualitativa aplicada e utilizou-se do método estudo de caso para analisar o objeto de pesquisa. $\mathrm{O}$ método em questão tem como característica analisar casos particulares que expressam uma realidade concreta.

Na visão de Flick (2009, p. 135), "O objetivo dos estudos de caso é a descrição exata ou a reconstrução de um caso." Assim sendo, objetivou-se descrever os registros bibliográficos selecionados para esta pesquisa como exemplos de casos relevantes ao tema em questão.

Goldenberg (2013, p. 33) afirma que "Este método supõe que se pode adquirir conhecimento do fenômeno estudado a partir da exploração intensa de um único caso.” Dessa forma, é possível aprofundar os conhecimentos acerca do objeto de estudo.

Os dois exemplos apresentados foram selecionados a partir do catálogo da UFSC (2015) e estão descritos de acordo com os campos e subcampos do formato Machine Readable Cataloging (MARC 21).

Efetuou-se um estudo comparativo entre os registros bibliográficos em AACR2 e em RDA selecionados com a finalidade de mostrar diferenças e semelhanças entre os elementos centrais da descrição. Outrossim, apresentaram-se, por meio de esquemas, os relacionamentos bibliográficos entre obras, expressões e/ou manifestações.

\section{APLICAÇÃO DO RDA EM REGISTROS BIBLIOGRÁFICOS}

Por meio de uma matriz de dados disponibilizada na página do RDA Toolkit (2010), são representadas as principais entidades e atributos de cada registro. $O$ modelo representativo dos relacionamentos entre as obras foi baseado no modelo proposto por Maxwell (2008, p. 84). Os dados destacados na segunda coluna representam os elementos novos acrescidos de acordo com o padrão RDA.

O primeiro exemplo apresentado no registro 1 é oriundo de uma dissertação da UFSC defendida em 2005 que abordou aspectos de uma obra (conhecida como "Cidade de Deus") cuja a manifestação em forma de livro (originado da dissertação de Paulo Lins) foi publicada em 1997 e, posteriormente, em 2002, foi transformada em filme. No Quadro 1 é possível verificar os elementos descritivos da referida dissertação. 
Quadro 1. Registro 1 em AACR2 e em RDA

\begin{tabular}{|c|c|}
\hline AACR2 & RDA \\
\hline $\begin{array}{l}100 \text { \$a Dutra, Eliane Aparecida } \\
245 \text { \$a Cidade de Deus \$b a banalização da violência } \\
\text { como discurso / \$c Eliane Aparecida Dutra ; } \\
\text { orientador, Pedro de Souza } \\
260 \text { \$c } 2005 \\
300 \text { \$a } 1 \text { v. ; \$c } 30 \text { cm } \\
502 \text { \$a Dissertação (mestrado) - Universidade Federal } \\
\text { de Santa Catarina, Centro de Comunicação e } \\
\text { Expressão, Programa de Pós-Graduação em Literatura, } \\
\text { Florianópolis, 2005 } \\
504 \text { \$a Inclui bibliografia } \\
650 \text { \$a Literatura } \\
650 \text { \$a Cinema e literatura } \\
650 \text { \$a Violência } \\
650 \text { \$a Criminalidade urbana } \\
650 \text { \$a Critica cinematográfica } \\
700 \text { \$a Souza, Pedro de, \$e orientador } \\
710 \text { \$a Universidade Federal de Santa Catarina. \$b } \\
\text { Programa de Pós-Graduação em Literatura } \\
85640 \text { \$u } \\
\text { <http://www.tede.ufsc.br/teses/PLIT0207.pdf> }\end{array}$ & 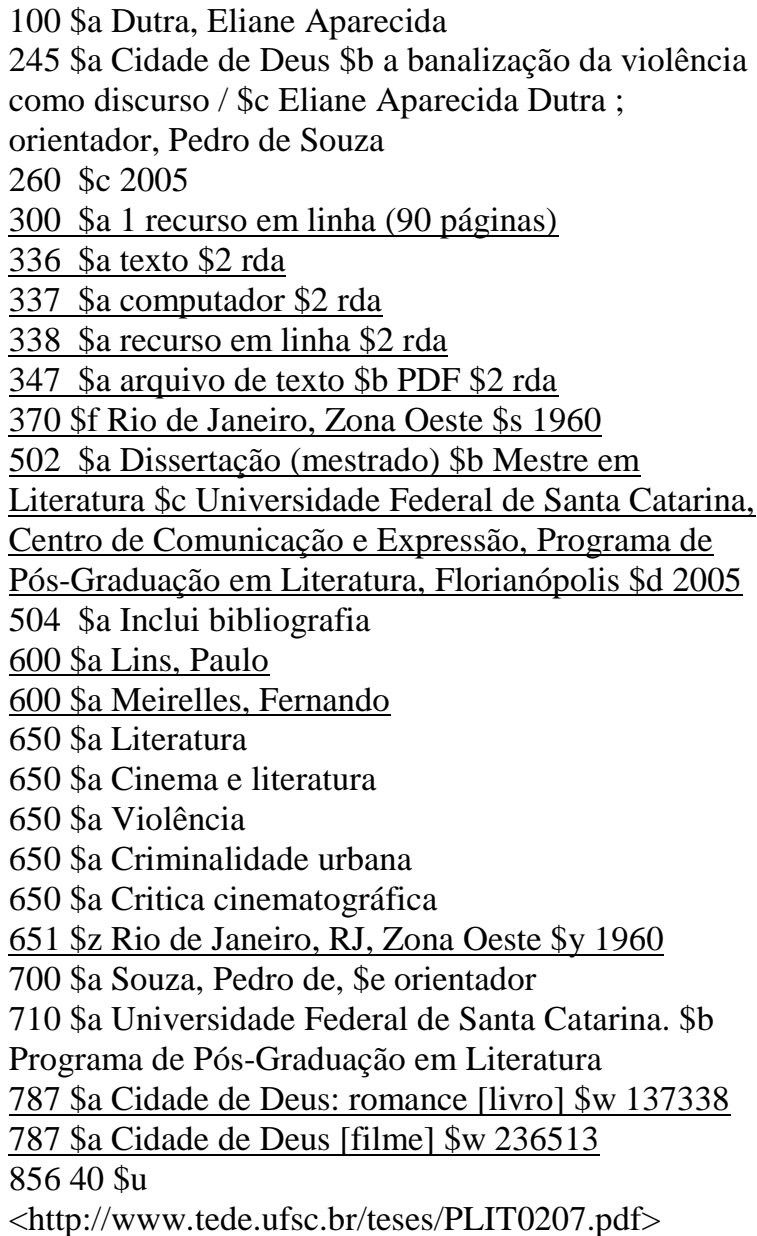 \\
\hline
\end{tabular}

Fonte: Elaborado pelas autoras (2015).

Quando comparados os registros bibliográficos das teses e das dissertações nos dois padrões em AACR2 e em RDA, visualiza-se que, de forma geral, a descrição não modificou muito, exceto pela inclusão de campos para descrição física de formato, mídia e suporte (campos 336, 337, 338 e 347), além de abarcar mais pontos de acesso temáticos.

Destaca-se que a extensão do item no campo 300 é diferente nos padrões por ter sido no registro em RDA considerada a numeração de páginas do arquivo PDF e, em AACR2, o número de páginas é do exemplar impresso. De acordo com o novo padrão, trata-se de duas expressões de uma mesma obra: o impresso e o eletrônico.

Surge, assim, a primeira questão: de que forma catalogar expressões diferentes de uma mesma obra? A sugestão, nesse caso, seria utilizar os dados de ambas as expressões (impressa e eletrônica) e informar, por exemplo, a extensão dos itens, constando duas paginações. No entanto, é possível que dados demais confundam o usuário do catálogo. 
No registro original em AACR2, o assunto principal abordado é sobre o tema "violência" na cidade do Rio de Janeiro na década de 1960. Assim, verificou-se que um local (Rio de Janeiro) e uma data não foram registrados como informação relevante dessas obras.

Na descrição bibliográfica, é possível a inclusão de um local geográfico como assunto no campo 651 do MARC 21 e também um complemento para a data. No registro em RDA, o campo 370 foi criado para acomodar um local e outras informações relacionadas à obra, incluindo o local de origem. Dessa maneira, foram incluídos esses campos para o local associado.

Outro ponto que se apresentou diferente é o campo 502, que diz respeito à nota específica para descrever trabalhos acadêmicos. Essa nota foi modificada no MARC 21 bibliográfico, pois foram desmembrados em subcampos para designar as informações a propósito da natureza do grau acadêmico, instituição que concede o título, entre outras. Compreende-se que esse desmembramento é pertinente, pois permite ao usuário acessar distintos pontos de acesso.

Utilizou-se o campo 787 para vincular no mesmo catálogo a obra do registro 1 com o livro e o filme. Destaca-se que o referido campo já existe no MARC 21 há algum tempo, diferentemente dos outros campos criados para acomodar as modificações do RDA, campos $336,337,338,347$ e 370 .

Também se incluiu como ponto de acesso o autor do livro (Paulo Lins) e o diretor do filme (Fernando Meirelles), responsáveis pelas obras analisadas. Na Figura 2, é possível visualizar como ocorreram os relacionamentos entre as obras analisadas do registro 1.

Figura 2. Relacionamentos do registro 1 


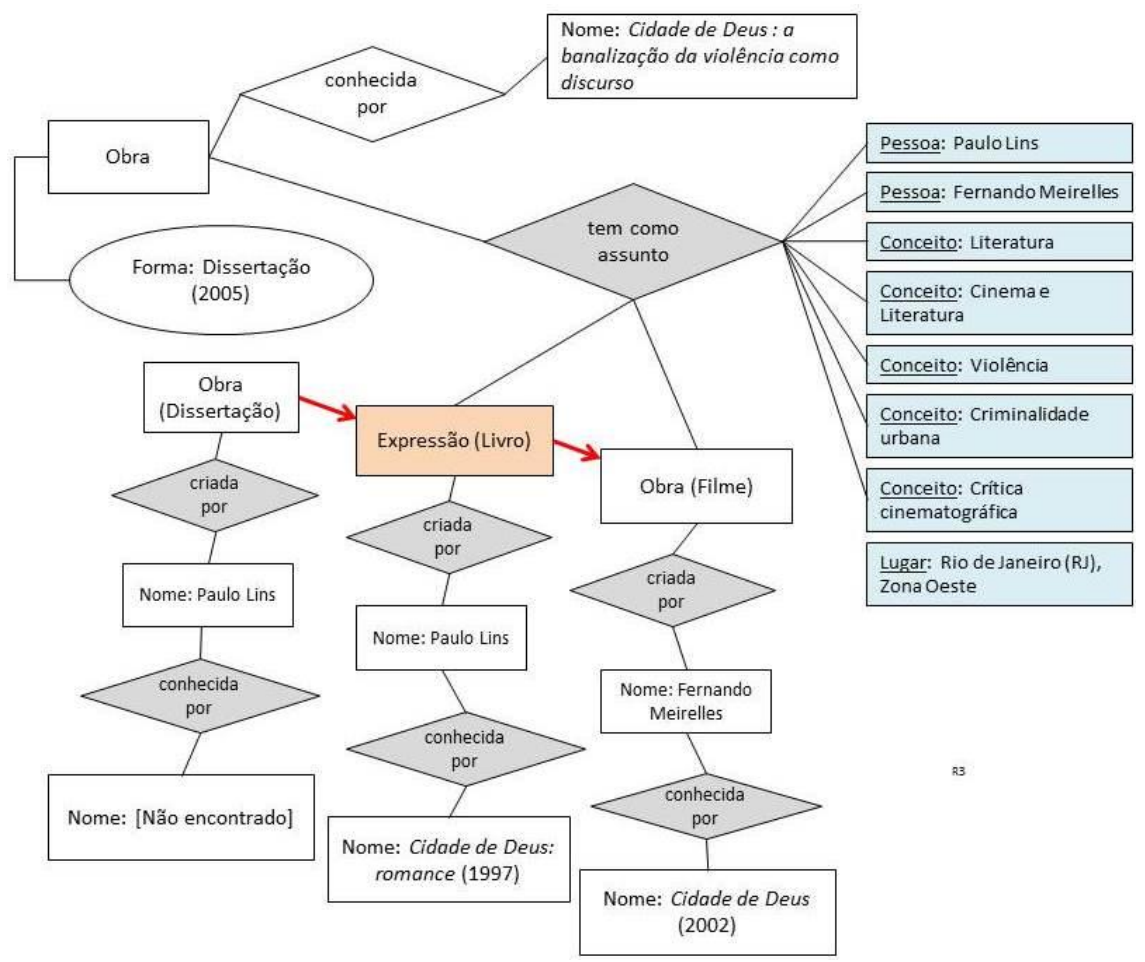

Fonte: Elaborado pelas autoras (2015).

Destaca-se que o livro que foi a temática do registro 1 é oriundo de uma outra dissertação de mestrado ${ }^{1}$, ou seja, o trabalho acadêmico do autor originou uma nova expressão no formato de livro, que posteriormente foi transformado em filme, conforme apontado pelas setas em vermelho. Esse trabalho acadêmico de Paulo Lins, mesmo não sendo o foco da análise do registro 1, foi apresentado na Figura 2 para expor como ocorreu o relacionamento, ainda que não tenha ligação de forma direta com a dissertação da UFSC descrita no registro 1.

O registro 2 descreve uma dissertação que analisa a figura de um personagem de um livro e que originou, posteriomente, dois filmes. A obra possui ainda um contexto histórico como pano de fundo da análise, como pode ser verificado no Quadro 2.

Quadro 2. Registro 2 em AACR2 e em RDA

\begin{tabular}{|l|l|}
\hline \multicolumn{1}{|c|}{ AARC2 } & \multicolumn{1}{c|}{ RDA } \\
\hline 100 \$a Dias, Jóe José & 100 \$a Dias, Jóe José \\
245 \$a Expandindo o olhar \$b das páginas literárias ao & 245 \$a Expandindo o olhar \$b das páginas literárias ao \\
cinema a caricatura do Jeca na expressão de Lobato e & cinema a caricatura do Jeca na expressão de Lobato e \\
Mazzaropi / \$c Jóe José Dias ; orientador, João & Mazzaropi / \$c Jóe José Dias ; orientador, João \\
Hernesto Werber & Hernesto Werber \\
260 \$c 2007 & 260 \$c 2007 \\
\hline
\end{tabular}

\footnotetext{
${ }^{1} \mathrm{Na}$ descrição do registro 1 no campo 520 - resumo (não incluído neste artigo) foi mencionada a dissertação de Paulo Lins, no entanto, o foco de análise da dissertação da UFSC foi apenas o livro e o filme. Não foram encontradas maiores informações a respeito da dissertação de Paulo Lins. Para mais detalhes, sugere-se consultar a obra em questão no seguinte link: 〈http://www.tede.ufsc.br/teses/PLIT0207.pdf >.
}

\begin{tabular}{|c|c|c|c|c|c|}
\hline (C) RDBCI: Rev. Digit. Bibliotecon. Cienc. Inf. & Campinas, SP & v. 15 & n. & p. $130-147$ & jan./abr. 2017 \\
\hline
\end{tabular}




\begin{tabular}{|c|c|}
\hline $\begin{array}{l}\text { Expressão, Programa de Pós-Graduação em Literatura, } \\
\text { Florianópolis, } 2007 \\
504 \text { \$a Inclui bibliografia } \\
600 \text { \$a Lobato, Monteiro, \$d 1882-1948 \$x Crítica e } \\
\text { interpretação } \\
600 \text { \$a Mazzaropi, Amácio, \$d 1912-1981 } \\
650 \text { \$a Literatura } \\
650 \text { \$a Literatura brasileira } \\
650 \text { \$a Cinema e literatura }\end{array}$ & 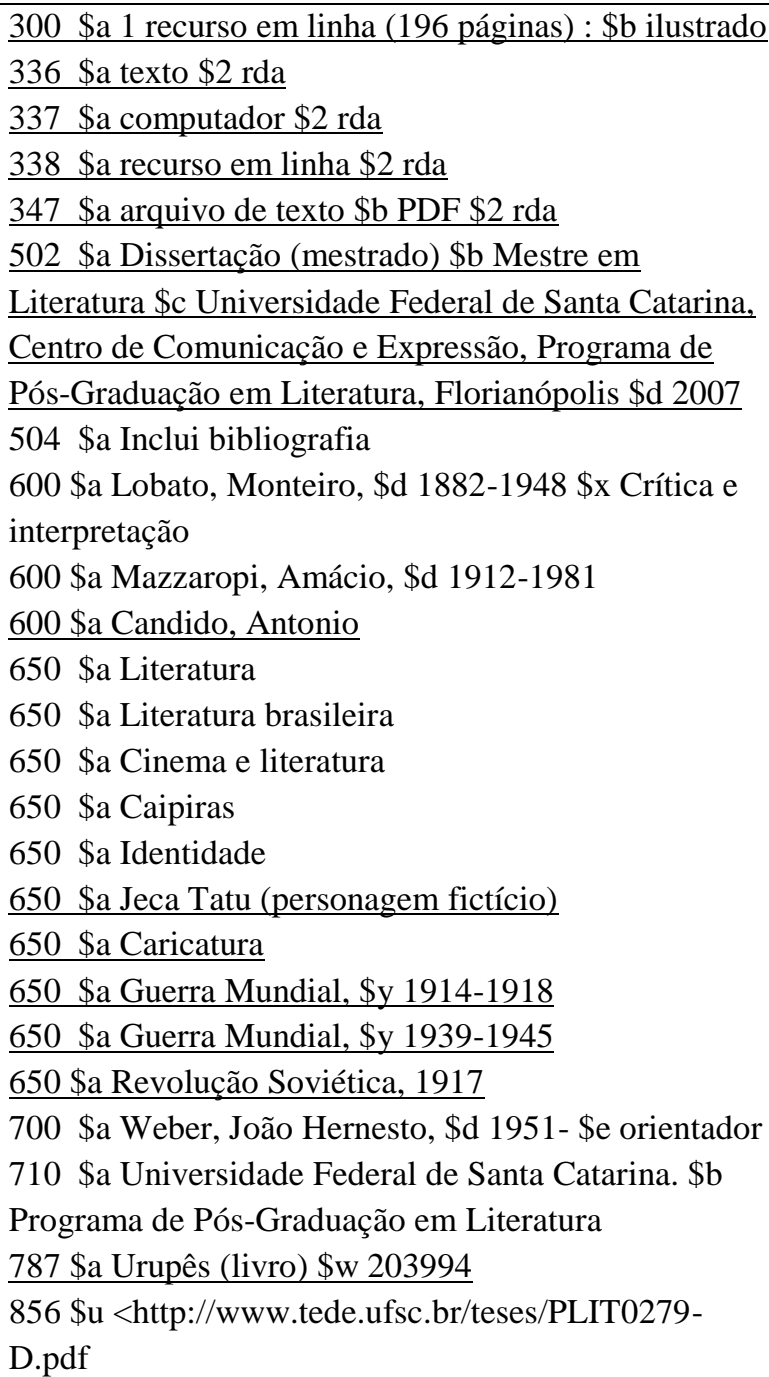 \\
\hline
\end{tabular}

Fonte: Elaborado pelas autoras (2015).

$\mathrm{Na}$ descrição física em RDA, considerou-se o número de páginas do arquivo PDF, diferentemente do registro original em AACR2, que levou em conta a extensão do item como volume.

Essa dissertação possui como pontos de acesso diversos elementos entre pessoas e conceitos. O principal tema é a figura caipira do personagem Jeca Tatu. Entre os temas descritos, incluíram-se os conceitos "Caricatura" e "Guerras Mundiais", além do autor "Antonio Candido", como pessoa relacionada.

A obra analisada (livro) foi vinculada ao respectivo registro por meio do campo 787 no catálogo da UFSC (2015). No entanto, os filmes citados não foram vinculados por não constar no mesmo catálogo. A Figura 3 apresenta os relacionamentos do registro 2.

Figura 3. Relacionamentos do registro 2 


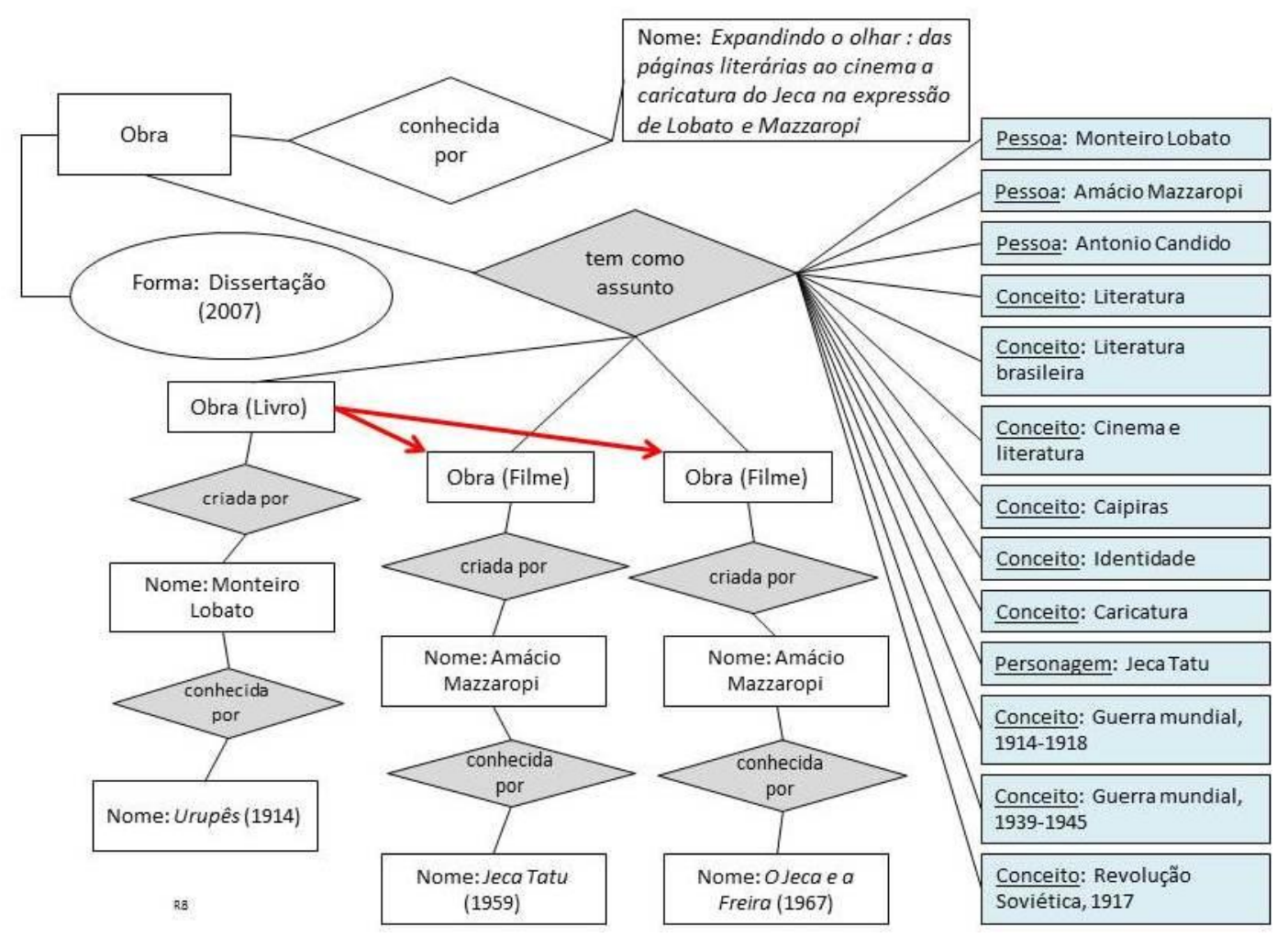

Fonte: Elaborado pelas autoras (2015).

A Figura 3 mostra que o livro originou a criação de outras duas obras no formato de filme, destacando o personagem de Jeca Tatu, indicadas pelas setas em vermelho.

O registro 2 possui diversos elementos associados, entre eles obras, pessoas, conceitos e datas. As obras vinculadas a esse registro possuem uma relação derivativa, assim como as pessoas associadas referem-se aos responsáveis pelas obras citadas. Por outro lado, os conceitos descritos no registro em RDA representam o conteúdo temático, e alguns, por sua vez, estão associados a datas históricas.

No presente estudo, constatou-se que o nível de detalhamento nos dois padrões apresenta diferenças relacionadas aos dados de formato, mídia e suporte. Ou seja, no registro em RDA, é possível incluir detalhes mais específicos para o acesso de um recurso, relacionando suas características peculiares.

Os relacionamentos entre as entidades são a parte mais rica dos modelos conceituais, pois permitem um dinamismo em catálogos de bibliotecas. Almendra, Gomes e Tolentino (2012) explicam que um catálogo construído nesse formato pode melhorar a recuperação da informação e a navegação pelo catálogo, o que ampliaria as opções ao usuário.

Para a implantação do novo padrão RDA, Santana Chavarria (2013) destaca que tal mudança implica novas exigências e investimentos de recursos de todos os tipos, especialmente econômicos, pois exige pessoal preparado e aparato tecnológico compatível. 


\section{CONSIDERAÇÕES FINAIS}

Foi realizado um estudo da estrutura e das orientações catalográficas recomendadas pelo RDA. É possível inferir pela estrutura da nova diretriz que há ainda questões em estudos para que as instruções estejam integralmente disponíveis para uso dos catalogadores, pois algumas seções no RDA Toolkit encontram-se em desenvolvimento.

As desvantagens identificadas na adoção desse novo padrão referem-se ao fato de ser o acesso ainda restrito, já que o RDA somente está disponível mediante o pagamento de uma assinatura. A outra desvantagem relaciona-se aos idiomas disponíveis uma vez que, durante a realização desta pesquisa, não estava disponível a versão em português. Contudo, recentemente, em março de 2015, foi disponibilizada a versão em espanhol, o que se torna uma opção aos bibliotecários brasileiros.

Com base na análise dos registros e dos resultados obtidos, conclui-se que o catalogador precisa despender mais atenção, conhecimento e tempo na sua prática. A aplicação do RDA exige mais atenção aos conceitos específicos do conteúdo (não só geral), mas também às pessoas e aos lugares.

A tarefa do catalogador pressupõe, ainda, realizar uma pesquisa mais ampla em outras fontes de informação sobre dados das obras em questão com vistas a identificar os relacionamentos entre obras.

Desse modo, infere-se que, com o maior detalhamento na aplicação do RDA, os registros sejam elaborados com mais dados, o que pode refletir melhorias na recuperação da informação, como também o reaproveitamento de dados, uma das mais significativas vantagens em se utilizar o novo padrão.

Por outro lado, as definições das entidades obra/expressão/manifestação como não estão totalmente claras, torna-se difícil definir quando uma obra (livro) que virou filme (homônimo) é uma nova obra, ou apenas uma nova expressão da mesma obra. Esse foi um obstáculo encontrado na elaboração dos registros, pois é complexo entender a lógica e o limite entre as entidades.

Conforme apontado nos resultados deste estudo, o RDA modificará os registros bibliográficos no tratamento de recursos principalmente nos dados relacionados ao acesso e à disponibilização do conteúdo. Significa que, para catalogar em RDA, é necessário especificar os detalhes sobre mídia, suporte e conteúdo.

Não obstante, precisa-se detalhar ao máximo os possíveis pontos de acesso do documento, incluindo conceitos, pessoas, lugares e outros, mostrando destacadamente os relacionamentos existentes entre entidades. 
O ponto mais impactante no tratamento descritivo e temático de um recurso informacional refere-se aos relacionamentos existentes entre as obras, não visualizados no atual catálogo da UFSC. As figuras construídas para representar o modelo entidaderelacionamento em cada registro permitiram identificar como uma obra relaciona-se com outra(s).

Os profissionais envolvidos nos setores de uma unidade de informação devem estar cientes das modificações acarretadas quando da implementação dos novos padrões. Consequentemente, a educação continuada do catalogador deve ser constante, acompanhando as tendências atuais de forma que o seu trabalho reflita na satisfação às necessidades informacionais dos usuários internos e externos do catálogo.

Destaca-se que, mesmo com todas as especificidades que o MARC 21 apresenta, referido formato não possibilita a total compatibilidade com o RDA, visto que os relacionamentos entre entidades não são totalmente identificados e descritos em um registro bibliográfico elaborado no MARC 21. Portanto, apresentam-se as seguintes sugestões para que futuramente seja adotado o novo padrão:

$\checkmark$ criar política institucional com critérios específicos para a catalogação em RDA;

$\checkmark$ solicitar aos desenvolvedores dos sistemas a inclusão dos campos criados para acomodar as inovações do novo padrão;

$\checkmark$ proporcionar treinamentos e capacitações à equipe envolvida; e

$\checkmark$ estabelecer parcerias com profissionais de outras instituições para debater questões sobre o RDA.

Além dessas propostas, considera-se essencial que os bibliotecários juntamente com outros profissionais, especialmente da área de computação, planejem e implementem sistemas eficientes que possam atender plenamente às tarefas dos usuários de catálogos. $\mathrm{O}$ novo perfil do catalogador exige que tal profissional tome decisões não só na elaboração dos registros, mas principalmente atuando como gestor na antecipação e execução de tarefas.

Sugerem-se, ao mesmo tempo, novas investigações acerca dessas questões, com destaque ao tratamento de recursos de outros tipos, como música ou peridiócos, além da implementação de um sistema informatizado que permita a atribuição de termos livres (tags/folksonomia) na representação temática de uma obra.

Esta pesquisa pode despertar interesse por outros estudos sobre o RDA e os modelos conceituais de modo que contribuam para a teoria na Ciência da Informação e da Biblioteconomia, como também para a prática bibliotecária. Os desdobramentos deste estudo poderão ainda sugerir novas investigações com outras abordagens e novos enfoques na análise de dados aplicando o código RDA. 


\section{ANÁLISIS DE PATRONES RDA: UN ESTUDIO APLICADO EN LAS TESIS Y DISERTACIONES EN LA LITERATURA Y EL CINE}

RESUMEN: La Descripción de Recursos y Acceso (RDA) es la nueva guía para la catalogación desarrollado para reemplazar a los anglo-americanos Reglas de Catalogación, segunda edición (AACR2), con el fin de mejorar la recuperación de la información. En este sentido, la presente investigación tiene como objetivo analizar cómo deben modificarse los registros bibliográficos de tesis y disertaciones de la literatura y de cine áreas con las normas del código de la RDA. La investigación cualitativa se caracteriza por ser aplicado exploratorio y descriptivo, y utiliza como un método de estudio de caso. Los resultados del estudio mostraron que la descripción bibliográfica presenta cambios significativos en relación con su formato, contenido y medios de un recurso. Con base en el análisis de los registros y los resultados obtenidos, se puede concluir que el catalogador tiene que gastar más atención, conocimiento y tiempo en su práctica, lo que sugiere que la necesidad de la educación profesional continua.

PALABRAS CLAVE: RDA. Descripción y acceso a recursos. La catalogación de Reglas. registro bibliográfico.

Submetido em: 28/04/2016

Aceito em: 10/11/2016

Publicado em: 17/11/2016

\section{REFERÊNCIAS}

ALMENDRA, Gabriela; GOMES, Jessica Nogueira; TOLENTINO, Vinicius de Souza. A análise do grupo 1 dos FRBRs na obra Gabriela, cravo e canela. In: ENCONTRO NACIONAL DE CATALOGADORES, 1.; ENCONTRO DE ESTUDOS E PESQUISAS EM CATALOGAÇÃO, 3., 2012, Rio de Janeiro. [Trabalhos publicados]... Rio de Janeiro: Biblioteca Nacional, 2012. Disponível em: <http://gepcat.blogspot.com.br/2012/10/i-enacate-iii-eepc-trabalhos.html>. Acesso em: 30 mar. 2015.

ASSUMPÇÃO, Fabrício Silva; SANTOS, Plácida Leopoldina Ventura Amorim da Costa. Resource Description and Access (RDA): objetivos, características e desenvolvimento do novo padrão para a descrição de recursos e acesso. In: CONGRESSO DE INICIAÇÃO CIENTÍFICA DA UNESP, 21., 2009, São José do Rio Preto. Trabalhos... São Paulo: UNESP, 2009. Disponível em: <http://prope.unesp.br/xxi_cic/27_33049772875.pdf>. Acesso em: 30 jan. 2015.

ASSUMPÇÃO, Fabrício Silva; SANTOS, Plácida Leopoldina Ventura Amorim da Costa. A utilização do Resource Description and Access (RDA) na criação de registros de autoridade para pessoas, famílias e entidades coletivas. Encontros Bibli, Florianópolis, v. 18, n. 37, p. 203-226, maio/ago. 2013. Disponível em: <http://dx.doi.org/10.5007/1518-

2924.2013v18n37p203>. Acesso em: 30 jan. 2015. 
CÓDIGO de Catalogação Anglo-Americano. 2. ed. São Paulo: Febab, 2005. 2 v.

DUTRA, Eliane Aparecida. Cidade de Deus: a banalização da violência como discurso. 2005. 1 v. Dissertação (Mestrado) - Universidade Federal de Santa Catarina, Centro de Comunicação e Expressão, Programa de Pós-Graduação em Literatura, Florianópolis, 2005.

FLICK, Uwe. Introdução à pesquisa qualitativa. 3. ed. Porto Alegre: Artmed, 2009.

GOLDENBERG, Mirian. A arte de pesquisar: como fazer pesquisa qualitativa em ciências sociais. 13. ed. Rio de Janeiro: Record, 2013.

INTERNATIONAL FEDERATION OF LIBRARY ASSOCIATIONS AND

INSTITUTIONS. Study Group on the Functional Requirements for Bibliographic

Records. Functional requirements for bibliographic records: final report. 2009.

Disponível em: <http://www.ifla.org/files/assets/cataloguing/frbr/frbr_2008.pdf>. Acesso em: 10 dez. 2013.

DIAS, Jóe José. Expandindo o olhar: das páginas literárias ao cinema a caricatura do Jeca na expressão de Lobato e Mazzaropi. 2007. 1 v. Dissertação (Mestrado) - Universidade Federal de Santa Catarina, Centro de Comunicação e Expressão, Programa de Pós-Graduação em Literatura, Florianópolis, 2007.

LE BOEUF, Patrick; TILLETT, Barbara; RIVA, Pat. O admirável mundo novo do FRBR. 2007. Disponível em:

$<$ http://www.imeicc5.com/download/portuguese/Presentations2c_BraveNewFRBRWorld(PR )_Port.pdf>. Acesso em: 3 mar. 2013.

MAXWELL, Robert L. FRBR: a guide for the perplexed. Chicago: American Library Association, 2008.

MEY, Eliane Serrão Alves; SILVEIRA, Naira. Christofoletti. Catalogação no plural. Brasília: Briquet de Lemos, 2009.

MORENO, Fernanda Passini; MEDEIROS, Marisa. Brascher. Basílio. Requisitos funcionais para registros bibliográficos - FRBR: um estudo no catálogo da rede bibliodata. Revista.

Ibero-Americana de Ciência da Informação, Brasília, v. 1, n. 1, p. 137-158, jan./jun. 2008.

MÜLLER, Adalberto. Além da literatura, aquém do cinema? Considerações sobre a intermidialidade. Outra Travessia, Florianópolis, n. 7, p. 47-53, 2008. Disponível em: <https://periodicos.ufsc.br/index.php/Outra/article/view/11974/11239>. Acesso em: 20 out. 2014.

OLIVER, Chris. Introdução à RDA: um guia básico. Brasília: Briquet de Lemos, 2011.

PICCO, Paola; ORTIZ REPISO, Virginia. RDA, el nuevo código de catalogación: cambios y desafíos para su aplicación. Revista Española de Documentación Científica, Madrid, v. 35, n. 1, p. 145-173, ene./mar. 2012.

RDA Toolkit: Resource Description \& Access. 2010. Disponível em: $<$ http://access.rdatoolkit.org/>. Acesso em: 16 mar. 2015.

\begin{tabular}{|c|c|c|c|c|c|}
\hline (C) RDBCI: Rev. Digit. Bibliotecon. Cienc. Inf. & Campinas, SP & v. 15 & n. & n. 130-147 & ian/abr 2017 \\
\hline
\end{tabular}


SANTANA CHAVARRIA, Evelia. Aplicación de RDA en la Biblioteca Nacional de México: avances y perspectivas. In: ENCONTRO INTERNACIONAL DE CATALOGADORES, 9.; ENCONTRO NACIONAL DE CATALOGADORES, 2., 2013, Rio de Janeiro. [Trabalhos publicados]... Rio de Janeiro: Biblioteca Nacional, 2013. Disponível em: <http://www.enacat.ufscar.br/index.php/eic-enacat/eic-enacat >. Acesso em: 24 mar. 2015.

TILLETT, Barbara. Requisitos funcionais para registros bibliográficos: o que é FRBR?: um modelo conceitual para o universo bibliográfico. Tradução: Lidia Alvarenga e Renato Rocha Souza. 2003. Disponível em: 〈http://www.loc.gov/catdir/cpso/o-que-e-frbr.pdf>. Acesso em: 15 ago. 2012.

UNIVERSIDADE FEDERAL DE SANTA CATARINA. [Catálogo eletrônico]. Disponível em: <http://150.162.1.90/pergamum/biblioteca/index.php>. Acesso em: 23 mar. 2015.

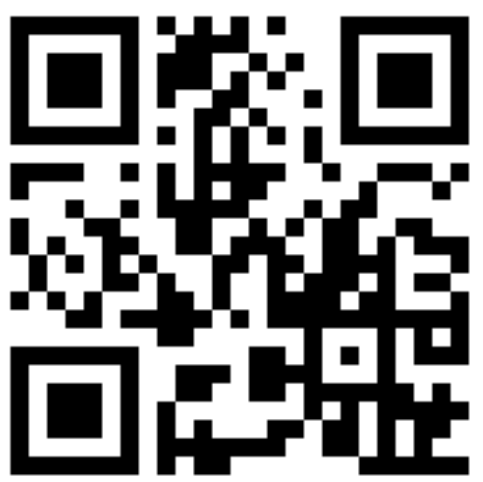

